\title{
The worst MitraClip Scenario: acute mitral regurgitation due to papillary muscle rupture
}

\author{
Davide Carino $^{1}$, Paolo Denti ${ }^{1}$, Alessandra Sala ${ }^{1}$, Wobbe Bouma ${ }^{2}$, Alessandro Castiglioni ${ }^{1}$, Ottavio Alfieri ${ }^{1}$, \\ Francesco Maisano ${ }^{1}$, Michele De Bonis ${ }^{1}$ \\ ${ }^{1}$ Department of Cardiac Surgery, IRCCS San Raffaele Scientific Institute, Vita-Salute San Raffaele University, Milan, Italy; ${ }^{2}$ Department of \\ Cardiothoracic Surgery, University Medical Center Groningen, Groningen, The Netherlands \\ Correspondence to: Davide Carino, MD. Department of Cardiac Surgery, IRCCS San Raffaele Scientific Institute, Vita-Salute San Raffaele University, \\ Via Olgettina 60, 20132, Milan, Italy. Email: carino.davide@hsr.it.
}

Submitted Jul 05, 2021. Accepted for publication Nov 23, 2021.

doi: 10.21037/acs-2021-ami-11

View this article at: https://dx.doi.org/10.21037/acs-2021-ami-11

\section{Clinical vignette}

An 81-year-old woman with a past medical history of coronary artery bypass grafting presented in the emergency department with increasing dyspnea and chest pain over the last two days. The electrocardiogram (ECG) showed ST segment elevation in the inferior leads, coupled with a high level of high-sensitivity cardiac troponin I. The patient was diagnosed with ST-segment elevation myocardial infarction. Coronary angiography was performed and showed an acute thrombosis of the right coronary artery in the middle portion, which was treated with percutaneous thromboaspiration and placement of two drug-eluting stents. Due to hemodynamic and respiratory deterioration, the patient was intubated, transferred to the intensive care unit (ICU) and an intra-aortic balloon pump (IABP) placed through the right femoral artery. Despite support with IABP and high doses of inotropes, the patient's hemodynamic situation further deteriorated. A transthoracic echocardiogram showed torrential mitral regurgitation (MR) due to a complete rupture of the postero-medial papillary muscle (PMR). The patient was deemed to have prohibitive surgical risk [estimated mortality: EuroSCORE II 71.02\%; Society of Thoracic Surgeons (STS) score 70.77\%] and after a multidisciplinary heart team discussion, an emergency MitraClip XTR (Abbott Structural Heart, Santa Clara, California, USA) was successfully performed. This case clearly shows that the MitraClip can be used as a good alternative in high-risk patients presenting with acute MR due to PMR.

\section{Surgical technique}

\section{Preparation}

The patient was transferred to the hybrid suite and prepped and draped for a MitraClip procedure, with both groins exposed.

\section{Exposure/access}

The right femoral vein was punctured at the groin. A 0.32 guidewire was advanced in the superior vena cava, and an 8.5 F, $63 \mathrm{~cm}$ long sheath (SL0TM; Abbott Laboratories, Abbott Park, IL, USA), with a dilator, was advanced over the guidewire. The Brockenbrough needle was inserted in the dilator and advanced until the tip of the needle was in the distal end of the dilator, but still inside it. The location of the trans-septal puncture is always of paramount importance for a smooth procedure, but in this case, due to the small size of the left atrium (LA) and the risk of impingement of the ruptured papillary muscle, the choice of the best site for the trans-septal puncture was even more important. 


\section{Operation}

The location for the trans-septal puncture was determined under transesophageal (TEE) guidance. The bicaval and aortic valve plane short-axis views were used to choose between a more superior or inferior (bicaval) and anterior or posterior (aortic short axis) puncture site. The final check before the puncture was obtained using a four-chamber view, evaluating the distance from the puncture, marked by septal tenting, to the free edge of the mitral valve at the site of regurgitation (puncture height). Due to the small size of the LA, it was difficult to have the puncture at a sufficient height; this made the procedure even more complex. Following the puncture, the trans-septal sheath was advanced in the LA. An Amplatz Super Stiff ${ }^{\mathrm{TM}} 0.035$ guidewire (Boston Scientific, Marlborough, MA, USA) and a $175-\mathrm{cm}$ standard J-Tip wire were then advanced into the left pulmonary vein. The trans-septal sheath was removed. Heparin was given with an activated clotting time target of 250-300 seconds. A 5-Fr pigtail catheter was advanced over the standard J-Tip wire, across the septum, into the LA for continuous pressure monitoring. The steerable guide catheter (SGC) of the MitraClip system and its dilator were advanced over the stiff wire across the septum. The dilator and the guidewire were removed, and the SCG was de-aired as usual. The clip delivery system (CDS) was positioned in the LA and the trajectory of the device was checked under TEE guidance using a combination of $\mathrm{X}$-plane and 3-D images. The delivery system was then advanced inside the left ventricle. The movement of the papillary muscle head complicated this part of the procedure. The grasping of the leaflets was accomplished with great care. The high heart rate, the risk of papillary impingement and the huge flail gap were all factors that significantly increased the difficulty of the procedure at that point. Although a significant reduction of MR was achieved with the first clip, we decided to implant a second clip medially. The deployment of the second clip was performed as previously described and due to the reduced flail gap, placement was less complex. After deployment of the second clip, MR was almost totally abolished with only trivial MR remaining.

\section{Completion}

The patient was transferred to the ICU with high inotropic support and IABP. The inotropes were slowly weaned, the
IABP removed and the patient extubated four days after the procedure.

\section{Comments}

Surgical outcomes for acute severe MR due to ischemic PMR have consistently been suboptimal, with high mortality rates $(1,2)$. In the presence of extremely high surgical risk, as in this case, a transcatheter approach may be considered as a reasonable rescue alternative to surgery. However, from a technical perspective, this procedure presents several challenging aspects. First and foremost, the reduced size of the LA significantly limits the height of the trans-septal puncture and the ability to correctly orient the clip. Second, the huge flail gap, the risk of papillary muscle impingement and the high heart rate, seriously complicate the grasping of the leaflets. Nevertheless, in centers with expertise in MitraClip implantation, this rescue procedure can be performed with good short-term results. Moreover, in case of a suboptimal result, MitraClip implantation does not exclude the possibility of performing surgical mitral valve replacement when the clinical condition of the patient improves.

\section{Acknowledgments}

Funding: None.

\section{Footnote}

Conflicts of Interest: The authors have no conflicts of interest to declare.

Open Access Statement: This is an Open Access article distributed in accordance with the Creative Commons Attribution-NonCommercial-NoDerivs 4.0 International License (CC BY-NC-ND 4.0), which permits the noncommercial replication and distribution of the article with the strict proviso that no changes or edits are made and the original work is properly cited (including links to both the formal publication through the relevant DOI and the license). See: https://creativecommons.org/licenses/by-nc-nd/4.0/.

\section{References}

1. Lorusso R, Gelsomino S, De Cicco G, et al. Mitral valve 
surgery in emergency for severe acute regurgitation: analysis of postoperative results from a multicentre study. Eur J Cardiothorac Surg 2008;33:573-82.

2. Gillinov AM, Wierup PN, Blackstone EH, et al.

Cite this article as: Carino D, Denti P, Sala A, Bouma W, Castiglioni A, Alfieri O, Maisano F, De Bonis M. The worst MitraClip Scenario: acute mitral regurgitation due to papillary muscle rupture. Ann Cardiothorac Surg 2022;11(3):340-342. doi: 10.21037/acs-2021-ami-11
Is repair preferable to replacement for ischemic mitral regurgitation? J Thorac Cardiovasc Surg 2001;122:1125-41. 